autoreactive B cell responses. Our aim was to compare basal activity and induced phosphorylation of AKT, ERK, p38 MAPK and CREB after stimulation of $B$ cells from RA patients and healthy individuals via $B C R$ and/or TLR9.

Materials and Methods Blood samples were collected from healthy donors and RA patients having moderate (DAS28 $3.2<5.1$ ) and active (DAS28 > 5.1) disease. B cells were stimulated with anti-Ig $(\mathrm{Fab})_{2}$ and/or CpG ODN. Naive and memory B cells were identified by anti-CD20-A647 and anti-CD27-PE Phosphorylation level of AKT, ERK, p38 and CREB was detected before and after the stimuli by specific phospho-antibodies using multiparameter phospho-flow analysis. Results were evaluated by the FlowJo software.

Results The basal level of phosphorylation of signalling molecules was significantly higher in RA patients as compared to healthy donors. The induced level of phosphorylation was also higher in RA samples in most of cases, CpG stimulated memory B cells from patients with active disease have shown the highest values. In antiIg plus CpG ODN stimulated samples phosphorylation of all molecules was significantly higher in both naïve and memory RA B cells as compared to healthy controls. However, when compared to unstimulated cells, the increment of phosphorylation in the stimulated cells was the same or lower in RA samples.

Conclusions We have shown differences in the activation state of AKT, ERK, p38 and CREB in B cells from healthy individuals and RA patients. The higher basal phosphorylation level indicates the activated state of RA B cells. The lower capability of activationinduced phosphorylation may be a result of lower responsiveness of RA B cells. The analysis of phosphorylation signature in RA B-cells may provide new information to a better understanding of the disease

\section{A5.24 NEUTROPHIL GRANULOCYTES RESPOND TO SURFACE-BOUND IMMUNE COMPLEXES CONTAINING ANTI-TYPE II COLLAGEN ANTIBODIES FROM RA PATIENTS}

doi:10.1136/annrheumdis-2013-203219.24

Vivek Anand Manivel, Mohammed Mullazehi, Azita Sohrabian, Barbro Persson, Amir Elshafie, Efstathios Kavvadas, Johan Rönnelid. Department of Immunology, Genetics and Pathology, Uppsala University, Uppsala, Sweden

Background and Objectives We have earlier shown that surfacebound immune complexes containing anti-type II collagen antibodies (anti-CII IC) from rheumatoid arthritis (RA) patients and anti-CII IC stimulate monocyte proinflammatory cytokine production, associated with an acute onset RA phenotype. Anti-CII IC in joint cartilage are exposed to cells in the synovial fluid (SF). Neutrophil granulocytes are the major cell type in SF, where they co-localise with mononuclear cells (MNC). The objective was to investigate whether also granulocytes respond to anti-CII IC, and whether such a response was dependent on interaction with other cells in SF.

Materials and Methods An anti-CII RA serum together with human native collagen (CII) was used to create surface-bound antiCII IC. Heparinised blood from 8 healthy donors was separated into neutrophil granulocytes ( $>95 \%$ purity) and MNC. For each donor, the granulocyte cell fractions as well as co-cultures (granulocytes + MNC) $(0.5 \times 10 \mathrm{E} 6 / \mathrm{ml}$ of each cell fraction) was cultured on anti-CII IC as well as on negative control IC prepared with normal human serum on CII and in a positive control IC system with purified IgG coated onto plastic. After 18 hours, cells were harvested for the measurement of CD11b, CD66b, CD16 and CD32 on granulocytes by flow cytometry, and supernatant levels of TNF and IL-8 was measured by ELISA.

Results In granulocyte cultures both anti-CII IC and control IC induced significant up-regulation of $\mathrm{CD} 11 \mathrm{~b}$ and $\mathrm{CD} 66 \mathrm{~b}$, and significant down-regulation of CD16 and CD32. When the granulocytes were co-cultured with MNC, there was a significant increase in CD11b up-regulation and CD16 down-regulation than granulocytes, with no effect on CD32 and CD66b. In the co-culture system, the anti-CII IC-induced production of IL-8 was significantly increased, but no such difference was noted for TNF. Isolated granulocyte fractions produced very low levels of TNF and IL-8 after IC stimulation.

Conclusions Isolated granulocytes respond to RA anti-CII IC in a model system mimicking IC in RA cartilage. The granulocyte responses depend on interaction with MNC.

Our anti-CII dependent RA phenotype is a human counterpart to collagen antibody-induced arthritis. Strong granulocyte reactivity to anti-CII IC might therefore be related to the Ncf1 gene involved in NADPH activity important in collagen-induced arthritis models.

\section{A5.25 PROTEOMIC ANALYSIS OF ANTI-CCP IMMUNOGLOBULINS FOR THE IDENTIFICATION OF RHEUMATOID ARTHRITIS PATIENTS THAT REQUIRE EARLY AGGRESSIVE TREATMENT REGIMENS}

doi:10.1136/annrheumdis-2013-203219.25

'Martijn M van Duijn, 'Theo M Luider, '2Johanna MW Hazes, ${ }^{2}$ Radboud JEM Dolhain. ${ }^{1}$ Department of Neurology, Erasmus MC, University Medical Center, Rotterdam, The Netherlands; '2Department of Rheumatology Erasmus MC, University Medical Center, Rotterdam, The Netherlands

Background and Objectives Antibodies against citrullinated peptides (ACPA) are a hallmark of rheumatoid arthritis (RA) patients and are associated with poor outcome. ACPA are usually detected using the anti-CCP test, identifying antibodies against a broad spectrum of citrullinated antigens. Most citrullinated antigens however appear to be bystander antigens that are not thought to be involved in the disease process. While controversy remains over the identity of the pathogenic antigen, it has been hypothesised that the fine-specificity of the ACPA response in the patient harbours prognostic properties. For this purpose sera will be studied from early RA patients using a novel technique that might reveal differences in that fine-specificity of the ACPA response.

Materials and Methods We recently showed that the antibody response to a particular antigen results in rearranged immunoglobulin segments that are shared between individuals exposed to that antigen. In patients with paraneoplastic neurological syndromes, such sequences were found after proteomics analysis of affinity enriched immunoglobulins. In the current study, ACPA positive RA sera were drawn from 58 patients participating in the tREACH-study. This is a study with a protocolised treatment regime for patients with early RA aimed at obtaining low-disease activity $(\mathrm{DAS}<2.4$ ). Poor outcome was defined as the need for treatment with anti-TNF to control disease activity. Sera were affinity enriched using CCP2 ELISA plates, and the specific IgG was analysed by LC-MS.

Results Up to $1 \mu \mathrm{g}$ of CCP specific IgG was obtained from $180 \mu \mathrm{l}$ of serum. The LC-MS data was analysed for correlations between ACPA derived peptides and the need for treatment with anti-TNF to control disease activity. However, no such correlations were found in excess of the false discovery rate in this dataset. In addition to ACPA, we also affinity purified rheumatoid factors from sera as a control. These preparations could be distinguished from the ACPA, suggesting our method performed appropriately.

Conclusions Our experiments could not show a significant difference between anti-CCP antibodies in early RA sera from patients with different disease outcome. While this could indicate that antiCCP fine-specificities are not associated with disease outcome, 